Bull. Korean Math. Soc. 51 (2014), No. 4, pp. 1115-1126

http://dx.doi.org/10.4134/BKMS.2014.51.4.1115

\title{
POINTWISE SLANT SUBMERSIONS
}

\author{
Jae Won Lee And Bayram SAhin
}

\begin{abstract}
The purpose of this paper is to study pointwise slant submersions from almost Hermitian manifolds which extends slant submersion in a natural way. Several basic results in this point of view are proven in this paper.
\end{abstract}

\section{Introduction}

As a generalization of slant submanifolds, pointwise slant submanifolds were first defined by Etayo [10] under the name of quasi-slant submanifolds and such submanifolds have been recently studied in details by Chen and Garay [7]. Chen and Garay obtain simple characterizations, give a method how to construct such submanifolds in Euclidean space and investigate geometric and topological properties of pointwise slant submanifolds. Since slant submanifolds include holomorphic submanifolds and totally real submanifolds, the class of pointwise slant submanifolds is a general notion for the theory of submanifolds of almost Hermitian manifolds.

On the other hand, Riemannian submersions between Riemannian manifolds were studied by O'Neill [19] and Gray [12]. Riemannian submersions have several applications in mathematical physics. Indeed, Riemannian submersions have their applications in the Yang-Mills theory ([2, 24]), Kaluza-Klein theory $([3,15])$, supergravity and superstring theories $([16,18])$, etc. Later such submersions were considered between manifolds with differentiable structures, see: [11]. As an analogue of holomorphic submanifolds, Watson defined almost Hermitian submersions between almost Hermitian manifolds and he showed that the base manifold and each fiber have the same kind of structure as the total space, in most cases [23]. We now recall the notion of almost Hermitian submersion. Let $M$ be a complex $m$-dimensional almost Hermitian manifold with Hermitian metric $g_{M}$ and almost complex structure $J_{M}$ and $N$ be a complex $n$ dimensional almost Hermitian manifold with Hermitian metric $g_{N}$ and almost complex structure $J_{N}$. A Riemannian submersion $F: M \longrightarrow N$ is called an

Received September 2, 2013.

2010 Mathematics Subject Classification. 53C15, 53B20, 53C43.

Key words and phrases. Riemannian submersion, Hermitian manifold, Kaehler manifold, pointwise slant submersion. 
almost Hermitian submersion (or holomorphic submersion) if $F$ is an almost complex mapping, i.e., $F_{*} J_{M}=J_{N} F_{*}$. The main result of this notion is that the vertical and horizontal distributions are $J_{M}$-invariant. On the other hand, Riemannian submersions from almost Hermitian manifolds onto Riemannian manifolds have been studied by many authors under the assumption that the vertical spaces of such submersions are invariant with respect to the complex structure. For instance, Escobales [9] studied Riemannian submersions from complex projective space onto a Riemannian manifold under the assumption that the fibers are connected, complex, totally geodesic submanifolds. One can see that this assumption implies that the vertical distribution is invariant. We note that almost Hermitian submersions have been extended to the almost contact manifolds [8], [13], locally conformal Kähler manifolds [17] and quaternion Kähler manifolds [14].

In [20], the second author introduced anti-invariant Riemannian submersions from almost Hermitian manifolds onto Riemannian manifolds as follows. Let $M$ be a complex $m$-dimensional almost Hermitian manifold with Hermitian metric $g_{M}$ and almost complex structure $J$ and $N$ be a Riemannian manifold with Riemannian metric $g_{N}$. Suppose that there exists a Riemannian submersion $F: M \longrightarrow N$ such that the integral manifold of the distribution ker $F_{*}$ is antiinvariant with respect to $J$, i.e., $J\left(\operatorname{ker} F_{*}\right) \subseteq\left(\operatorname{ker} F_{*}\right)^{\perp}$. Then we say that $F$ is an anti-invariant Riemannian submersion. Recently, the second author also introduced the notion of slant submersions from almost Hermitian manifolds onto arbitrary Riemannian manifolds [21] as follows: Let $F$ be a Riemannian submersion from an almost Hermitian manifold $\left(M_{1}, g_{1}, J_{1}\right)$ onto a Riemannian manifold $\left(M_{2}, g_{2}\right)$. If for any non-zero vector $X \in \Gamma\left(\operatorname{ker} F_{*}\right)$, the angle $\theta(X)$ between $J X$ and the space $\operatorname{ker} F_{*}$ is a constant, i.e. it is independent of the choice of the point $p \in M_{1}$ and choice of the tangent vector $X$ in $\operatorname{ker} F_{*}$, then we say that $F$ is a slant submersion. In this case, the angle $\theta$ is called the slant angle of the slant submersion.

In this paper, we study pointwise slant submersions from almost Hermitian manifolds which include slant submersions. As a result of this, they also cover holomorphic submersions and anti-invariant submersions [20]. In Section 2, we recall basic facts for Riemannian submersions and almost Hermitian manifolds. In Section 3, we define pointwise slant Riemannian submersions and give a method for obtaining examples of such submersions. We also obtain a characterization of pointwise slant submersions and check the relation between slant submanifolds and pointwise slant submanifolds. We also investigate the harmonicity of such maps and find necessary and sufficient conditions for the fibers to be totally geodesic submanifolds. Then we give necessary and sufficient conditions for pointwise slant Riemannian submersions to be totally geodesic. 


\section{Preliminaries}

Let $(\bar{M}, g, J)$ be an almost Hermitian manifold. This means that $\bar{M}$ admits a tensor field $J$ of type $(1,1)$ on $\bar{M}$ such that

$$
J^{2}=-I, \quad g(J X, J Y)=g(X, Y), \quad X, Y \in \Gamma(T \bar{M}) .
$$

An almost Hermitian manifold $\bar{M}$ is called Kähler manifold if

$$
\left(\bar{\nabla}_{X} J\right) Y=0, \quad X, Y \in \Gamma(T \bar{M}),
$$

where $\bar{\nabla}$ is the operator of Levi-Civita covariant differentiation.

Let $\left(M^{m}, g_{M}\right)$ and $\left(N^{n}, g_{N}\right)$ be Riemannian manifolds, where $\operatorname{dim} M=m$, $\operatorname{dim} N=n$ and $m>n$. A Riemannian submersion $F: M \longrightarrow N$ is a map from $M$ onto $N$ satisfying the following axioms:

(S1) $F$ has the maximal rank.

(S2) The differential $F_{*}$ preserves the lengths of horizontal vectors.

For each $q \in N, F^{-1}(q)$ is an $(m-n)$-dimensional submanifold of $M$. The submanifolds $F^{-1}(q)$ are called fibers. A vector field on $M$ is called vertical if it is always tangent to fibers. A vector field on $M$ is called horizontal if it is always orthogonal to fibers. A vector field $X$ on $M$ is called basic if $X$ is horizontal and $F$-related to a vector field $X_{*}$ on $N$, i.e., $F_{*} X_{p}=X_{* F(p)}$ for all $p \in M$. Note that we denote the projection morphisms on the distributions ker $F_{*}$ and $\left(\operatorname{ker} F_{*}\right)^{\perp}$ by $\mathcal{V}$ and $\mathcal{H}$, respectively.

We recall the following lemma from O'Neil [19].

Lemma 2.1. Let $F: M \longrightarrow N$ be a Riemannian submersion between Riemannian manifolds and $X, Y$ be basic vector fields of $M$. Then

(a) $g_{M}(X, Y)=g_{N}\left(X_{*}, Y_{*}\right) \circ F$.

(b) the horizontal part $[X, Y]^{\mathcal{H}}$ of $[X, Y]$ is a basic vector field and corresponds to $\left[X_{*}, Y_{*}\right]$, i.e., $F_{*}([X, Y])=\left[X_{*}, Y_{*}\right]$.

(c) $[V, X]$ is vertical for any vector field $V$ of $\operatorname{ker} F_{*}$.

(d) $\left(\nabla_{X}^{M} Y\right)^{\mathcal{H}}$ is the basic vector field corresponding to $\nabla_{X_{*}}^{N} Y_{*}$.

The geometry of Riemannian submersions is characterized by O'Neil's tensors $\mathcal{T}$ and $\mathcal{A}$ defined for vector fields $E, F$ on $M$ by

$$
\begin{aligned}
\mathcal{A}_{E} F & =\mathcal{H} \nabla_{\mathcal{H} E} \mathcal{V} F+\mathcal{V} \nabla_{\mathcal{H} E} \mathcal{H} F, \\
\mathcal{T}_{E} F & =\mathcal{H} \nabla_{\mathcal{V}_{E}} \mathcal{V} F+\mathcal{V} \nabla_{\mathcal{V}_{E}} \mathcal{H} F,
\end{aligned}
$$

where $\nabla$ is the Levi-Civita connection of $g_{M}$. It is easy to see that a Riemannian submersion $F: M \longrightarrow N$ has totally geodesic fibers if and only if $\mathcal{T}$ vanishes identically. For any $E \in \Gamma(T M), \mathcal{T}$ is vertical, $\mathcal{T}_{E}=\mathcal{T}_{\mathcal{V} E}$ and $\mathcal{A}$ is horizontal, $\mathcal{A}_{E}=\mathcal{A}_{\mathcal{H} E}$. We note that the tensor $\mathcal{T}$ and $\mathcal{A}$ satisfy

$$
\begin{aligned}
& \mathcal{T}_{U} W=\mathcal{T}_{W} U, \quad U, W \in \Gamma\left(\operatorname{ker} F_{*}\right), \\
& \mathcal{A}_{X} Y=-\mathcal{A}_{Y} X=\frac{1}{2} \mathcal{V}[X, Y], \quad X, Y \in \Gamma\left(\left(\operatorname{ker} F_{*}\right)^{\perp}\right) .
\end{aligned}
$$


On the other hand, from (2.3) and (2.4), we have

$$
\begin{aligned}
\nabla_{V} W & =\mathcal{T}_{V} W+\hat{\nabla}_{V} W, \\
\nabla_{V} X & =\mathcal{H} \nabla_{V} X+\mathcal{T}_{V} X, \\
\nabla_{X} V & =\mathcal{A}_{X} V+\mathcal{V} \nabla_{X} V, \\
\nabla_{X} Y & =\mathcal{H} \nabla_{X} Y+\mathcal{A}_{X} Y
\end{aligned}
$$

for $X, Y \in \Gamma\left(\left(\operatorname{ker} F_{*}\right)^{\perp}\right)$ and $V, W \in \Gamma\left(\operatorname{ker} F_{*}\right)$, where $\hat{\nabla}_{V} W=\mathcal{V} \nabla_{V} W$. If $X$ is basic, then $\mathcal{H} \nabla_{V} X=\mathcal{A}_{X} V$. Note that we will also use the symbols $\mathcal{V}$ and $\mathcal{H}$ to denote the distributions $\operatorname{ker} F_{*}$ and $\left(\operatorname{ker} F_{*}\right)^{\perp}$, respectively.

Finally, we recall the notion of harmonic maps between Riemannian manifolds. Let $\left(M, g_{M}\right)$ and $\left(N, g_{N}\right)$ be Riemannian manifolds and supposed that $F: M \longrightarrow N$ is a smooth map. Then the differential $F_{*}$ of $F$ can be viewed a section of the bundle $\operatorname{Hom}\left(T M, F^{-1} T N\right) \rightarrow M$, where $F^{-1} T N$ is the pullback bundle which has fibers $\left(F^{-1} T N\right)_{p}=T_{F(p)} N, p \in M$. Hom $\left(T M, F^{-1} T N\right)$ has a connection $\nabla$ induced from the Levi-Civita connection $\nabla^{M}$ and the pullback connection $\nabla^{F}$. Then the second fundamental form of $F$ is given by

$$
\left(\nabla F_{*}\right)(X, Y)=\nabla_{X}^{F}\left(F_{*}(Y)\right)-F_{*}\left(\nabla_{X}^{M} Y\right)
$$

for $X, Y \in \Gamma(T M)$. It is known that the second fundamental form is symmetric. We note that if $F$ is a Riemannian submersion between Riemannian manifolds, then we have

$$
\left(\nabla F_{*}\right)(X, Y)=0
$$

for $X, Y \in \Gamma\left(\left(\operatorname{ker} F_{*}\right)^{\perp}\right)$. A smooth map $F:\left(M, g_{M}\right) \longrightarrow\left(N, g_{N}\right)$ is said to be harmonic if $\operatorname{trace}\left(\nabla F_{*}\right)=0$. On the other hand, the tension field of $F$ is the $\operatorname{section} \tau(F)$ of $\Gamma\left(F^{-1} T N\right)$ defined by

$$
\tau(F)=\operatorname{div} F_{*}=\sum_{i=1}^{m}\left(\nabla F_{*}\right)\left(e_{i}, e_{i}\right),
$$

where $\left\{e_{1}, \ldots, e_{m}\right\}$ is the orthonormal frame on $M$. Then it follows that $F$ is harmonic if and only if $\tau(F)=0$ (for details, see [1]).

\section{Pointwise slant submersions}

In this section, we are going to define pointwise slant submersions and investigate the geometry of such submersions.

Definition 3.1. Let $F$ be a Riemannian submersion from an almost Hermitian manifold $\left(M, g_{M}, J\right)$ onto a Riemannian manifold $\left(N, g_{N}\right)$. If, at each given point $p \in M$, the Wirtinger angle $\theta(X)$ between $J X$ and the space $\left(\operatorname{ker} F_{*}\right)_{p}$ is independent of the choice of the nonzero vector $X \in\left(\operatorname{ker} F_{*}\right)$, then we say that $F$ is a pointwise slant submersion. In this case, the angle $\theta$ can be regarded as a function on $M$, which is called the slant function of the pointwise slant submersion. 
Definition 3.2. A point $p$ in a pointwise slant submersion is called totally real if its slant function $\theta=\frac{\pi}{2}$ at $p$. Similarly, a point $p$ is called a complex point if its slant function $\theta=0$ at $p$.

Note. A pointwise slant submersion is called slant in the sense of [21] if its slant function $\theta$ is globally constant, i.e., $\theta$ is also independent of the choice of the point on $M$. In this case, the constant $\theta$ is called the slant angle of the slant submersion. A pointwise slant submersion $F$ is called totally real if every point of $M$ is a totally real point.

We now give an example for pointwise slant submersions.

Example 3.1. Let $\left(\mathbb{R}^{4}, g_{0}\right)$ be the standard Euclidean space with the standard metric $g_{0}$. Consider $\left\{J_{0}, J_{1}\right\}$ a pair of almost complex structures on $\mathbb{R}^{4}$ satisfying $J_{0} J_{1}=-J_{1} J_{0}$, where

$$
\begin{aligned}
& J_{0}(a, b, c, d)=(-c,-d, a, b), \\
& J_{1}(a, b, c, d)=(-b, a, d,-c) .
\end{aligned}
$$

For any real-valued function $f: \mathbb{R}^{4} \longrightarrow \mathbb{R}$, we define new almost complex structure $J_{f}$ on $\mathbb{R}^{4}$ by

$$
J_{f}=(\cos f) J_{0}+(\sin f) J_{1} .
$$

Then $\mathbb{R}_{f}^{4}=\left(\mathbb{R}^{4}, J_{f}, g_{0}\right)$ is an almost Hermitian manifold.

Consider a Riemannian submersion $F: \mathbb{R}_{f}^{4} \longrightarrow \mathbb{R}^{2}$ by $F\left(x_{1}, x_{2}, x_{3}, x_{4}\right)=$ $\left(\frac{x_{1}-x_{2}}{\sqrt{2}}, \frac{x_{3}-x_{4}}{\sqrt{2}}\right)$. Then $F$ is a pointwise slant submersion with the slant function $f$.

Note. In fact, as Chen and Garay emphasized in [7] for pointwise slant submanifolds, if a Riemannian submersion is invariant [22] or holomorphic [23] with respect to $J_{1}$, then it will always produce a pointwise slant submersion with the slant function $f$. This shows that there are many pointwise slant submersions.

Now, we assume that $F$ is a Pointwise slant Riemannian submersion from an almost Hermitian manifold $\left(M, g_{M}, J\right)$ onto Riemannian manifold $\left(N, g_{N}\right)$. Then for $V \in \Gamma\left(\operatorname{ker} F_{*}\right)$, we have

$$
J V=\varphi V+\omega V,
$$

where $\varphi V(\omega V$, resp.) is vertical (horizontal, resp.) of $J V$. Also for $X \in$ $\Gamma\left(\left(\operatorname{ker} F_{*}\right)^{\perp}\right)$, we have

$$
J X=\mathcal{B} X+\mathcal{C} X
$$

where $\mathcal{B} X(\mathcal{C} X$, resp.) is vertical (horizontal, resp.) of $J X$. We denote the orthogonal complementary distribution to $\omega\left(\operatorname{ker} F_{*}\right)$ in $\left(\operatorname{ker} F_{*}\right)^{\perp}$ by $\mu$. That is,

$$
\left(\operatorname{ker} F_{*}\right)^{\perp}=\omega\left(\operatorname{ker} F_{*}\right) \oplus \mu \text {. }
$$


It is easy to see that $\mu$ is an invariant subbundle of $\left(\operatorname{ker} F_{*}\right)^{\perp}$ with respect to $J$. Using (2.7), (2.8), (3.1) and (3.2), we obtain

$$
\begin{aligned}
& \left(\nabla_{V} \omega\right) W=\mathcal{C} \mathcal{T}_{V} W-\mathcal{T}_{V} \varphi W, \\
& \left(\nabla_{V} \varphi\right) W=\mathcal{B} \mathcal{T}_{V} W-\mathcal{T}_{V} \omega W,
\end{aligned}
$$

where $\nabla$ is the Levi-Civita connection on $M$ and

$$
\begin{aligned}
& \left(\nabla_{V} \omega\right) W:=\mathcal{H} \nabla_{V} \omega W-\omega \hat{\nabla}_{V} W, \\
& \left(\nabla_{V} \varphi\right) W:=\hat{\nabla}_{V} \varphi W-\varphi \hat{\nabla}_{V} W
\end{aligned}
$$

for $V, W \in \Gamma\left(\operatorname{ker} F_{*}\right)$. We say that $\omega$ is parallel with respect to the Levi-Civita connection $\nabla$ on ker $F_{*}$ if its covariant derivative with respect to $\nabla$ vanishes, i.e., $\left(\nabla_{V} \omega\right) W=0$ for $V, W \in \Gamma\left(\operatorname{ker} F_{*}\right)$.

The proof of the following result is the same as slant immersions (see [7]), therefore we omit its proof.

Theorem 3.1. Let $F$ be a Riemannian submersion from an almost Hermitian manifold $\left(M, g_{M}, J\right)$ onto a Riemannian manifold $\left(N, g_{N}\right)$. Then $F$ is a pointwise slant submersion if and only if there exists a real-valued function $\theta$ defined on $\operatorname{ker} F_{*}$ such that

$$
\varphi^{2}=-\left(\cos ^{2} \theta\right) I .
$$

A pointwise slant submersion is said to be proper if it has neither complex points nor totally real points.

Corollary 3.2. Let $F$ be a pointwise proper slant submersion from an almost Hermitian manifold $\left(M^{m}, g_{M}, J\right)$ onto a Riemannian manifold $\left(N^{n}, g_{N}\right)$ with the slant function $\theta$. If $e_{1}, e_{2}, \ldots, e_{m-n}$ are locally orthonormal basis for $\operatorname{ker} F_{*}$, then

$$
\left\{\csc \theta \omega e_{1}, \csc \theta \omega e_{2}, \ldots, \csc \theta \omega e_{m-n}\right\}
$$

is a local orthonormal basis of $\omega\left(\operatorname{ker} F_{*}\right)$.

The following result is a consequence from Theorem 3.2 and Corollary 3.3.

Corollary 3.3. Let $F$ be a pointwise proper slant submersion from an almost Hermitian manifold $\left(M, g_{M}, J\right)$ onto a Riemannian manifold $\left(N, g_{N}\right)$. Then the distributions $\mu$ and $\operatorname{ker} F_{*} \oplus \omega\left(\operatorname{ker} F_{*}\right)$ are even dimensional.

The following proposition gives a characterization of pointwise slant submersions

Proposition 3.4. $F$ is a pointwise slant submersion from an almost Hermitian manifold $\left(M, g_{M}, J\right)$ onto a Riemannian manifold $\left(N, g_{N}\right)$ if and only if $\varphi$ : ker $F_{*} \longrightarrow \operatorname{ker} F_{*}$ preserves orthogonality, $\varphi$ carries each pair of orthogonal vectors into orthogonal vectors. 
Proof. Denote by $S\left(\operatorname{ker} F_{*}\right)$ the unit kernel bundle of ker $F_{*}$. Then consider the function $\theta: S\left(\operatorname{ker} F_{*}\right) \longrightarrow R$ on the unit kernel bundle $S\left(\operatorname{ker} F_{*}\right)$. With respect to the induced metric, $S_{p}\left(\operatorname{ker} F_{*}\right)$ is the unit sphere in $\operatorname{ker} F_{* p}$ centered at $o$.

At a given point $p \in M$, we have

$$
g_{M}(\varphi X, \varphi X)=\cos ^{2} \theta(X)
$$

for any unit vector $X \in \operatorname{ker} F_{* p}$. For each unit vector $Y$ tangent to $\operatorname{ker} F_{* p}$ at $X \in \operatorname{ker} F_{* p}(X \perp Y)$, using $g_{M}\left(\varphi X,\left(\nabla_{Y} \varphi\right) X\right)=0$, we have

$$
\begin{aligned}
2 g_{M}(\varphi X, \varphi Y) & =2 g_{M}\left(\varphi X, \varphi\left(\nabla_{Y} X\right)\right)=2 g_{M}\left(\varphi X, \nabla_{Y}(\varphi X)-\left(\nabla_{Y} \varphi\right) X\right) \\
& =2 g_{M}\left(\varphi X, \nabla_{Y}(\varphi X)\right)=Y g_{M}(\varphi X, \varphi X)=-(Y \theta) \sin 2 \theta(X) .
\end{aligned}
$$

In the long run, $\varphi$ carries each pair of orthogonal vector in ker $F_{* p}$ into a pair of orthogonal vectors in $\operatorname{ker} F_{* p}$ if and only if the slant function $\theta$ is independent of $X \in \operatorname{ker} F_{* p}$.

Proposition 3.5. Let $F$ be a pointwise slant submersion from a Kähler manifold $\left(M, g_{M}, J\right)$ onto a Riemannian manifold $\left(N, g_{N}\right)$. $F$ is slant submersion if and only if

for $X \in \Gamma\left(\operatorname{ker} F_{*}\right)$.

$$
\mathcal{T}_{X} \omega \varphi X=\mathcal{T}_{\varphi X} \omega X
$$

Proof. Let $F$ be a pointwise slant submersion from a Kähler manifold $\left(M, g_{M}, J\right)$ onto a Riemannian manifold $\left(N, g_{N}\right)$ with the slant function $\theta$. For any unit vector field $X \in \Gamma\left(\operatorname{ker} F_{*}\right)$, we may put

$$
\varphi X=(\cos \theta) X^{*}
$$

where $X^{*}$ is a unit vector field orthogonal of $X$. Then for $Y \in \Gamma\left(\operatorname{ker} F_{*}\right)$, we have

$$
\begin{aligned}
\nabla_{Y}(J X)= & \nabla_{Y}\left((\cos \theta) X^{*}\right)+\nabla_{Y}(\omega X) \\
= & -(\sin \theta)(Y \theta) X^{*}+(\cos \theta)\left(\mathcal{T}_{Y} X^{*}+\hat{\nabla}_{Y} X^{*}\right) \\
& +\mathcal{H} \nabla_{Y}(\omega X)+\mathcal{T}_{Y}(\omega X) .
\end{aligned}
$$

On the other hand, we also have

$$
\begin{aligned}
\nabla_{Y}(J X) & =J\left(\nabla_{Y} X\right) \\
& =\mathcal{B} \mathcal{T}_{Y} X+\mathcal{C} \mathcal{T}_{Y} X+\varphi \hat{\nabla}_{Y} X+\omega \hat{\nabla}_{Y} X .
\end{aligned}
$$

Comparing the vertical components of (3.5) and (3.6)

$$
-(\sin \theta)(Y \theta) X^{*}+(\cos \theta) \hat{\nabla}_{Y} X^{*}+\mathcal{T}_{Y}(\omega X)=\mathcal{B} \mathcal{T}_{Y} X+\varphi \hat{\nabla}_{Y} X .
$$

Therefore, by taking the inner product of (3.7) with $X^{*}$, we have

$$
-(\sin \theta)(Y \theta)+g_{M}\left(\mathcal{T}_{X *} \omega X, Y\right)=g_{M}\left(Y, \mathcal{T}_{X} \omega X^{*}\right)
$$

Consequently, the pointwise slant sumbersion is slant if and only if

$$
\mathcal{T}_{X} \omega X=\mathcal{T}_{X} \omega X^{*}
$$


Corollary 3.6. Let $F$ be a pointwise slant submersion from a Kähler manifold $\left(M, g_{M}, J\right)$ onto a Riemannian manifold $\left(N, g_{N}\right)$. If $F$ has totally geodesic fibers, it is slant.

Proof. If $F$ has totally geodesic fibers, $\mathcal{T}$ vanishes identically. Thus the proof follows from Proposition 3.6.

Let $F$ be a Remannian submersion from a Riemannian manifold $\left(M, g_{M}\right)$ onto a Riemannian manifold $\left(N, g_{N}\right)$. Recall that a Riemannian submersion is called a Riemannian submersion with totally umbilical fibers if

$$
\mathcal{T}_{X} Y=g_{M}(X, Y) H
$$

for $X, Y \in \Gamma\left(\operatorname{ker} F_{*}\right)$, where $H$ is the mean curvature vector field of the fiber.

Corollary 3.7. Let $F$ be a pointwise proper slant submersion with totally umbilical fibers from a Kähler manifold $\left(M^{2 n}, g_{M}, J\right)$ onto a Riemannian manifold $\left(N^{n}, g_{N}\right)$. If $F$ has no totally geodesic fibers, then it is always non-slant.

Proof. Assume that $F$ is a pointwise proper slant submersion with totally umbilical fibers. Then we have

$$
\mathcal{T}_{X} Y=g_{M}(X, Y) H
$$

for $X, Y \in \Gamma\left(\operatorname{ker} F_{*}\right)$. Therefore, we have

$$
\begin{aligned}
g_{M}\left(\mathcal{T}_{X} \omega \varphi X, Y\right) & =-g_{M}(X, Y) g_{M}(\omega \varphi X, H), \\
g_{M}\left(\mathcal{T}_{\varphi X} \omega X, Y\right) & =-g_{M}(\varphi X, Y) g_{M}(\omega X, H) .
\end{aligned}
$$

If the submersion is slant, then Proposition 3.6 and (3.8)-(3.9) imply that

$$
g_{M}(\omega \varphi X, H) X=g_{M}(\omega X, H) \varphi X .
$$

Since $g_{M}(X, \varphi X)=0$,

$$
g_{M}(\omega \varphi X, H)=g_{M}(\omega X, H)=0,
$$

which implies $H \in \Gamma(\mu)$. On the other hand, we note that $\operatorname{dim}\left(\operatorname{ker} F_{*}\right)=$ $2 n-n=n$. Thus using Corollary 3.3, we have $\operatorname{dim}\left(\left(\operatorname{ker} F_{*}\right) \oplus \omega\left(\operatorname{ker} F_{*}\right)\right)=$ $2(2 n-n)=2 n$. Therefore, we get $\operatorname{dim}(\mu)=2 n-2 n=0$, which contradicts that $H$ vanishes identically.

We now investigate certain properties of pointwise slant submersions. The following theorem proposes a new condition for Riemannian submersions to be harmonic. We first recall that if $F:\left(M_{1}, g_{1}\right) \longrightarrow\left(M_{2}, g_{2}\right)$ is a map between Riemannian manifolds $\left(M_{1}, g_{1}\right)$ and $\left(M_{2}, g_{2}\right)$, then the adjoint map ${ }^{*} F_{*}$ of $F_{*}$ is characterized by $g_{1}\left(x,{ }^{*} F_{* p_{1}} y\right)=g_{2}\left(F_{* p_{1}} x, y\right)$ for $x \in T_{p_{1}} M_{1}, y \in T_{F\left(p_{1}\right)} M_{2}$ and $p_{1} \in M_{1}$. Considering $F_{*}^{h}$ at each $p_{1} \in M_{1}$ from a linear transformation

$$
F_{* p_{1}}^{h}:\left(\left(\operatorname{ker} F_{*}\right)^{\perp}\left(p_{1}\right), g_{1 p_{1}\left(\left(\operatorname{ker} F_{*}\right)^{\perp}\left(p_{1}\right)\right)}\right) \longrightarrow\left(\operatorname{range}_{*}\left(p_{2}\right), g_{\left.2 p_{2}\left(\operatorname{range}_{*}\right)\left(p_{2}\right)\right)}\right),
$$


we will denote the adjoint of $F_{*}^{h}$ by ${ }^{*} F^{h}{ }_{* p_{1}}$. Let ${ }^{*} F_{* p_{1}}$ be the adjoint of $F_{* p_{1}}$ : $\left(T_{p_{1}} M_{1}, g_{p_{p_{1}}}\right) \longrightarrow\left(T_{p_{2}} M_{2}, g_{2_{p_{2}}}\right)$. Then the linear transformation

$$
\left({ }^{*} F_{* p_{1}}\right)^{h}: \operatorname{range}_{*}\left(p_{2}\right) \longrightarrow\left(\operatorname{ker} F_{*}\right)^{\perp}\left(p_{1}\right)
$$

defined by $\left({ }^{*} F_{* p_{1}}\right)^{h} y={ }^{*} F_{* p_{1}} y$, where $y \in \Gamma\left(r a n g e F_{* p_{1}}\right), p_{2}=F\left(p_{1}\right)$, is an isomorphism and $\left(F_{* p_{1}}^{h}\right)^{-1}=\left({ }^{*} F_{* p_{1}}\right)^{h}={ }^{*}\left(F_{* p_{1}}^{h}\right)$.

Theorem 3.8. Let $F$ be a pointwise slant submersion from Kaehler manifold $\left(M_{1}, g_{1}, J\right)$ onto a Riemannian manifold $\left(M_{2}, g_{2}\right)$ and have no complex points. Then $F$ is harmonic if and only if

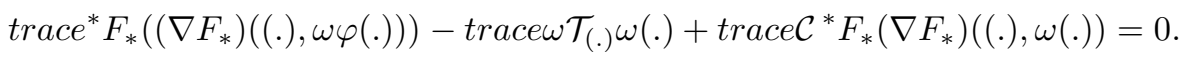

Proof. From (2.7), (2.1), (2.2), (3.1) and (3.2) we have

$$
g_{1}\left(\mathcal{T}_{U} U, X\right)=-g_{1}\left(\nabla_{U} J \varphi U, X\right)+g_{1}\left(\nabla_{U} \omega U, J X\right)
$$

for $U \in \Gamma(\mathcal{V})$ and $X \in \Gamma(\mathcal{H})$. Then Theorem 3.1, (3.1) and (2.8) imply

$$
\begin{aligned}
g_{1}\left(\mathcal{T}_{U} U, X\right)= & -g_{1}(\sin 2 \theta U(\theta) U, X)+g_{1}\left(\cos ^{2} \theta \nabla_{U} U, X\right) \\
& -g_{1}\left(\nabla_{U} \omega \varphi U, X\right)+g_{1}\left(\mathcal{T}_{U} \omega U, \mathcal{B} X\right) \\
& +g_{1}\left(\nabla_{U} \omega U, \mathcal{C} X\right) .
\end{aligned}
$$

Using (2.11) we arrive at

$$
\begin{aligned}
\sin ^{2} \theta g_{1}\left(\mathcal{T}_{U} U, X\right)= & g_{2}\left(\left(\nabla F_{*}\right)(U, \omega \varphi U), F_{*}(X)\right)+g_{1}\left(\mathcal{T}_{U} \omega U, \mathcal{B} X\right) \\
& -g_{2}\left(\left(\nabla F_{*}\right)(U, \omega U), F_{*}(\mathcal{C} X)\right) .
\end{aligned}
$$

Then from (3.2) we get

$$
\begin{aligned}
\sin ^{2} \theta g_{1}\left(\mathcal{T}_{U} U, X\right)= & g_{1}\left({ }^{*} F_{*}\left(\left(\nabla F_{*}\right)(U, \omega \varphi U)\right), X\right)-g_{1}\left(\omega \mathcal{T}_{U} \omega U, X\right) \\
& +g_{1}\left(\mathcal{C}^{*} F_{*}\left(\left(\nabla F_{*}\right)(U, \omega U)\right), X\right) .
\end{aligned}
$$

Thus the proof follows from a fact that a Riemannian submersion is harmonic if and only if its fibers are minimal submanifolds in $M_{1}$.

Next result gives a necessary and sufficient condition for fibers of a pointwise slant submersion to be totally geodesic submanifolds.

Theorem 3.9. Let $F$ be a pointwise slant submersion from a Kaehler manifold $\left(M_{1}, g_{1}, J\right)$ onto a Riemannian manifold $\left(M_{2}, g_{2}\right)$. Then the fibers are totally geodesic submanifolds in $M_{1}$ if and only if

$$
\begin{aligned}
g_{2}\left(\nabla_{X^{\prime}}^{2} F_{*}(\omega U), F_{*}(\omega V)\right)= & -\sin ^{2} \theta g_{1}([U, X], V)+\sin 2 \theta X(\theta) g_{1}(U, V) \\
& +g_{1}\left(\mathcal{A}_{X} \omega \varphi U, V\right)-g_{1}\left(\mathcal{A}_{X} \omega U, \varphi V\right),
\end{aligned}
$$

where $X$ and $X^{\prime}$ are $F$-related vector fields and $\nabla^{2}$ is the Levi-Civita connection on $M_{2}$. 
Proof. Since $\mathcal{T}$ is skew symmetric with respect to $g_{1}$ and $\nabla$ is a torsion free connection on $M_{1}$, using (2.8) we have

$$
g_{1}\left(\mathcal{T}_{U} V, X\right)=-g_{1}([U, X], V)-g_{1}\left(\nabla_{X} U, V\right)
$$

for $U, V \in \Gamma(\mathcal{V})$ and $X \in \Gamma(\mathcal{H})$. Then from (3.1), (3.2), (2.1) and (2.2) we obtain

$$
\begin{aligned}
g_{1}\left(\mathcal{T}_{U} V, X\right)= & -g_{1}([U, X], V)+g_{1}\left(\nabla_{X} \varphi^{2} U+\omega \varphi U, V\right) \\
& -g_{1}\left(\nabla_{X} \omega U, \varphi V\right)-g_{1}\left(\nabla_{X} \omega U, \omega V\right) .
\end{aligned}
$$

Thus Theorem 3.1, (2.10) and (2.12) imply

$$
\begin{aligned}
g_{1}\left(\mathcal{T}_{U} V, X\right)= & -g_{1}([U, X], V)+\sin 2 \theta X(\theta) g_{1}(U, V)-\cos ^{2} \theta g_{1}\left(\nabla_{X} U, V\right) \\
& +g_{1}\left(\mathcal{A}_{X} \omega \varphi U, V\right)-g_{1}\left(\mathcal{A}_{X} \omega U, \varphi V\right) \\
& -g_{2}\left(\nabla_{X^{\prime}}^{2} F_{*}(\omega U), F_{*}(\omega V)\right) .
\end{aligned}
$$

Since $\nabla$ is a torsion free connection, using skew symmetry property of $\mathcal{T}$ and (2.7) we get

$$
\begin{aligned}
\sin ^{2} \theta g_{1}\left(\mathcal{T}_{U} V, X\right)= & -\sin ^{2} \theta g_{1}([U, X], V)+\sin 2 \theta X(\theta) g_{1}(U, V) \\
& +g_{1}\left(\mathcal{A}_{X} \omega \varphi U, V\right)-g_{1}\left(\mathcal{A}_{X} \omega U, \varphi V\right) \\
& -g_{2}\left(\nabla_{X^{\prime}}^{2} F_{*}(\omega U), F_{*}(\omega V)\right)
\end{aligned}
$$

which gives the assertion.

Finally we give a result for pointwise slant submersions to be totally geodesic maps. We recall that a differentiable map $F$ between Riemannian manifolds $\left(M_{1}, g_{1}\right)$ and $\left(M_{2}, g_{2}\right)$ is called a totally geodesic map if $\left(\nabla F_{*}\right)(X, Y)=0$ for all $X, Y \in \Gamma\left(T M_{1}\right)$. A geometric interpretation of a totally geodesic map is that it maps every geodesic in the total manifold into a geodesic in the base manifold in proportion to arc lengths.

Theorem 3.10. Let $F$ be a pointwise slant submersion from Kaehler manifold $\left(M_{1}, g_{1}, J\right)$ onto a Riemannian manifold $\left(M_{2}, g_{2}\right)$. Then $F$ is a totally geodesic map if and only if

$$
\begin{aligned}
g_{2}\left(\nabla_{X^{\prime}}^{2} F_{*}(\omega U), F_{*}(\omega V)\right)= & -\sin ^{2} \theta g_{1}([U, X], V)+\sin 2 \theta X(\theta) g_{1}(U, V) \\
& +g_{1}\left(\mathcal{A}_{X} \omega \varphi U, V\right)-g_{1}\left(\mathcal{A}_{X} \omega U, \varphi V\right)
\end{aligned}
$$

and

$$
g_{1}\left(\mathcal{A}_{X} \omega U, \mathcal{B} Y\right)=-\left[-g_{2}\left(\nabla_{X}^{F} F_{*}(\omega \varphi U), F_{*}(Y)\right)+g_{2}\left(\nabla_{X}^{F} F_{*}(\omega U), F_{*}(\mathcal{C} Y)\right)\right],
$$

where $X$ and $X^{\prime}$ are $F$-related vector fields and $\nabla^{F}$ is the pull-back connection along $F$. 
Proof. By definition, it follows that $F$ is totally geodesic if and only if $\left(\nabla F_{*}\right)(X$, $Y)=0$ for $X, Y \in \Gamma(\mathcal{H}),\left(\nabla F_{*}\right)(X, V)=0$ for $V \in \Gamma(\mathcal{V})$ and $\left(\nabla F_{*}\right)(U, V)=0$ for $U \in \Gamma(\mathcal{V})$. From $(2.12)$, it follows that $\left(\nabla F_{*}\right)(X, Y)=0$. Also from Theorem 3.9, it follows that $\left(\nabla F_{*}\right)(U, V)=0$ if and only if $(3.10)$ is satisfied.

On the other hand, since $F$ is a Riemannian submersion, using (2.11) we have

$$
g_{2}\left(\left(\nabla F_{*}\right)(X, U), F_{*}(Y)\right)=-g_{1}\left(\nabla_{X} U, Y\right) .
$$

Then from Theorem 3.1, (2.1), (2.2), (3.1), (3.2) and (2.10) we obtain

$$
\begin{aligned}
g_{2}\left(\left(\nabla F_{*}\right)(X, U), F_{*}(Y)\right)= & -\sin 2 \theta X(\theta) g_{1}(U, Y)-\cos ^{2} \theta g_{1}\left(\nabla_{X} U, Y\right) \\
& +g_{1}\left(\nabla_{X} \omega \varphi U, Y\right)-g_{1}\left(\mathcal{A}_{X} \omega U, \mathcal{B} Y\right) \\
& -g_{1}\left(\nabla_{X} \omega U, \mathcal{C} Y\right) .
\end{aligned}
$$

Thus using (2.11) and (2.12) we get

$$
\begin{aligned}
& \sin ^{2} \theta g_{2}\left(\left(\nabla F_{*}\right)(X, U), F_{*}(Y)\right) \\
= & -\left[-g_{2}\left(\nabla_{X}^{F} F_{*}(\omega \varphi U), F_{*}(Y)\right)+g_{2}\left(\nabla_{X}^{F} F_{*}(\omega U), F_{*}(\mathcal{C} Y)\right)\right] \\
& -g_{1}\left(\mathcal{A}_{X} \omega U, \mathcal{B} Y\right)
\end{aligned}
$$

which completes proof.

\section{References}

[1] P. Baird and J. C. Wood, Harmonic Morphisms Between Riemannian Manifolds, London Mathematical Society Monographs, 29, Oxford University Press, The Clarendon Press, Oxford, 2003.

[2] J. P. Bourguinon and H. B. Lowson, Stability and isolation phenomena for Yang-Mills fields, Comm. Math. Phys. 79 (1981), no. 2, 189-230.

[3] _ A mathematician's visit to Kaluza-Klein theory, Rend. Sem. Mat. Univ. Politec. Torino (1989), Special Issue, 143-163.

[4] J. L. Cabrerizo, A. Carriazo, and M. Fernandez, Slant submanifolds in Sasakian manifolds, Glasg. Math. J. 42 (2000), no. 1, 125-138.

[5] B. Y. Chen, Slant Immersions, Bull. Austral. Math. Soc. 41 (1990), no. 1, 134-147.

[6] _ Geometry of Slant Submanifolds, Katholieke Universiteit Leuven, Leuven, 1990.

[7] B. Y. Chen and O. Garay, Pointwise slant submanifolds in almost Hermitian manifolds, Turk. J. Math. 36 (2012), no. 4, 630-640.

[8] C. Chinea, Almost contact metric submersions, Rend. Circ. Mat. Palermo 43 (1985), no. $1,89-104$

[9] R. H. Escobales Jr., Riemannian submersions from complex projective space, J. Differential Geom. 13 (1978), no. 1, 93-107.

[10] F. Etayo, On quasi-slant submanifolds of an almost Hermitian manifold, Publ. Math. Debrecen 53 (1998), no. 1-2, 217-223.

[11] M. Falcitelli, S. Ianus, and A. M. Pastore, Riemannian Submersions and Related Topics, World Scientific, River Edge, NJ, 2004.

[12] A. Gray, Pseudo-Riemannian almost product manifolds and submersion, J. Math. Mech. 16 (1967), 715-737.

[13] S. Ianus, A. M. Ionescu, R. Mocanu, and G. E. Vilcu, Riemannian submersions from almost contact metric manifolds, Abh. Math. Semin. Univ. Hambg. 81 (2011), no. 1, $101-114$. 
[14] S. Ianus, R. Mazzocco, and G. E. Vilcu, Riemannian submersions from quaternionic manifolds, Acta Appl. Math. 104 (2008), no. 1, 83-89.

[15] S. Ianus and M. Visinescu, Kaluza-Klein theory with scalar fields and generalised Hopf manifolds, Classical Quantum Gravity 4 (1987), no. 5, 1317-1325.

[16] _ Space-time compactification and Riemannian submersion, The mathematical heritage of C. F. Gauss, 358-371, World Sci. Publ., River Edge, NY, 1991.

[17] J. C. Marrero and J. Rocha, Locally conformal Kähler submersions, Geom. Dedicata 53 (1994), no. 3, 271-289.

[18] M. T. Mustafa, Applications of harmonic morphisms to gravity, J. Math. Phys. 41 (2000), no. 10, 6918-6929

[19] B. O'Neill, The fundamental equations of a submersion, Mich. Math. J. 13 (1966), 458-469.

[20] B. Sahin, Anti-invariant Riemannian submersions from almost Hermitian manifolds, Cent. Eur. J. Math. 8 (2010), no. 3, 437-447.

[21] Slant submersions from almost hermitian manifolds, Bull. Math. Soc. Sci. Math. Roumanie 54(102) (2011), no. 1 93-105.

[22] _ Riemannian submersions from almost Hermitian manifolds, Taiwaneese J. Math. 17 (2013), no. 2, 629-659.

[23] B. Watson, Almost Hermitian submersions, J. Differential Geometry 11 (1976), no. 1, $147-165$.

[24] — $, G, G$ '-Riemannian submersioins and nonlinear gauge field equations of general relativity, Global analysis-analysis on manifolds, 324-349, Teubner-Texte Math., 57 Teubner, Leipzig, 1983.

JAE WON LEE

School of General Education

YEUNGNAM UNIVERSITY

Gyengsan 712-749, Korea

E-mail address: leejaew@yu.ac.kr

BAYRAM SAHIN

Department of Mathematics

INONU UNIVERSITY

Malatya 44280, TURKey

E-mail address: bayram.sahin@inonu.edu.tr 